\title{
Mechanical Properties of Polyester Toughened with Nano-Silica
}

\author{
${ }^{1}$ Diyar Kaka*, ${ }^{2}$ Roma A. Fatah, ${ }^{2}$ Parzhin Gharib, ${ }^{2}$ Ahmad Mustafa \\ ${ }^{1}$ Department of Petroleum and Energy Engineering, Sulaymani Polytechnic University, Sulaymaniya - Iraq \\ ${ }^{2}$ Department of Manufacturing Engineering, Koya University, Erbil - Iraq
}

\section{Article information}

Article history:

Received: August, 15, 2021

Accepted: October, 21, 2021

Available online: December, 14, 2021

Keywords:

Nanocomposite,

RVE,

Nano particles

*Corresponding Author:

Diyar Kaka

diyar.kaka@spu.edu.iq

DOI:

https://doi.org/10.53523/ijoirVol8I3ID67

\begin{abstract}
The fabrication of nanocomposites has played role to the development of the nanotechnology and the technology of advanced composite materials. Thermoset polymers are used in engineering applications widely. Their mechanical properties can be change with adding particles. The mechanism of toughening polymers has been suggested recently by reinforcing well dispersed particles to the plain polymer. Nano-silica particles were added to thermoset polymer of polyester to evaluate their influence on the mechanical properties of the toughened polymer using both experimental and numerical methods. The Representative Volume Element (RVE) approach, which employs finite element models, has been developed to achieve that aim numerically for various types of nano-particle reinforcement ratios. In each case, the stiffness has been calculated with using the equivalent homogeneous material concept. Experimentally, toughened thermoset polymers of polyester reinforced with nano-silica were prepared with different particle content ratio. Several tests were conducted on the nanocomposite, and it was observed increasing nano-silica ratio caused increase in Young's modulus and decrease in ductility.
\end{abstract}

\section{Introduction}

In the last decade, the field of polymer nanocomposites has exhibited development in the advanced composite materials engineering. The nano-composites of particles reinforcement to a polymer matrix is one of the attractive fields. That kind of reinforcement improves some mechanical properties in different applications. Several products that made of polymer and need improving in some mechanical properties can be reinforced with particles, this is called toughened polymer. Toughened polymer has become interest of different fields of industry in automobile and airplanes [1]. The particles of nano-Silica normally available in forms of white amorphous powder, fine or colloid suspension. The important characteristic of those particles are the large surface area and the smooth nonporous surface. This can enhance the strong physical contact when reinforced in polymers. Nowadays silica particles are commercially available in all sizes ranging from nanometer to micrometer [2]. Polyesters are a family of polyesters that consist of alkyl thermoset resins and it is characterized by vinyl unsaturation.

In the end of the last century, it was discovered that epoxy resins could be reinforced and toughened by the addition of a rigid thermoplastic phase of particles, thus the rigid-rigid polymer alloy concept was born [3]. The 
use of inorganic fillers of particles and reinforcement to modify the properties of polymers has been around for many years [4,5]. Jumahat et.al [6] found that the addition of nano-silica improved the tensile properties of the epoxy. They added $13 \mathrm{wt} \%$ nano-silica into the epoxy matrix and they observed enhancing the tensile modulus by $21 \%$, tensile strength by $20 \%$ and failure strain by $10 \%$. Also, they added maximum reinforcement of nanosilica to the epoxy with value of $(25 \mathrm{wt} \%)$ to give an observable increase in the strength and the tensile Young's modulus about $38 \%$ and $24 \%$, respectively, by comparing with plain polymer. This suggests that the nano-filler-matrix interaction is very strong therefore the nanocomposites exhibited higher strength compared to the pristine polymer. Zappalorto et. al [7] were noted that nanocomposites pose higher tensile strengths and Young's modulus than the plain epoxy without reducing its failure strain significantly, even at high nanosilica content. They observed that nanocomposites and resist more loads by comparing with the plain epoxy and contribute to obtain larger tensile modulus and strength properties. Raymond [8] measured the Young's modulus and yield stress at different values of weight fractions. The results exhibited increase in the Young's modulus and the yield stress for nanocomposite of epoxy/silica, and it increased gradually with the content of silica particles. Wang et .al [9] reinforced epoxy with nano-silica nanoparticles with various content of nanosilica by 0wt.\%, 1wt.\%, 3wt.\%, 5wt.\%, 7 wt.\%. It was found the modulus improvement to $15.67 \%, 31.05 \%, 32.68 \%$, $33.66 \%$, respectively, and the failure improvement to $3.33 \%, 26.96 \%, 78.19 \%, 14.19 \%$, respectively compared with pure epoxy resin [9]. The role of the particle morphology has been studied by a number of researchers, and it was found that the effect of multi-layered core-shell latex particles on toughening PMMA [10].

Several Researchers have studied the micromechanical models of the composite materials [11-15]. Azmi [16] were used micromechanical model with representative element methods RVE to find the mechanical properties of the composite materials. So, they used a numerical methodology, implemented in Abaqus is based on the representative volume element (RVE) concept. They reinforced about $10 \%$ content of nanosilica to the resin and they noted improving the Young's modulus of the plain resin about $40 \%$. It was observed the presence of a graded interphase around the reinforcing particles and interaction effects play an important role and need to be accounted for the prediction of the elastic modulus [16, 17]. He et. al [18] have modelled two different shaped particles reinforcement and studied their effects on the elastic mechanical properties of composites. They noted increase in the Young's modulus of the composites about $150 \%$ than that of matrix for the case of strong interface [18].

From the review, it can observe that different kinds of polymers were reinforced with different types of particles by using experimental method and numerical method. However, there is ambiguity with adding nanosilica to polyester polymer. This project concentrates on effect of adding particles of nano-silica to the mechanical properties of thermoset polymer of polyester. Also, The use of new methodology of calculating mechanical properties of heterogeneous materials such representative volume element (RVE).

\section{Material Processing and Testing}

\subsection{Preparation of Nanocomposite Nanosilica-Polyester}

The polyester is polymer contain the ester functional group in every repeat unit of their main chain. As a specific material, it most commonly refers to a type called polyethylene terephthalate (PET). The nanoparticles of silica were obtained from Wacker Company in Germany. Based on the manufacturer, their particle size was between $50 \mathrm{~nm}-100 \mathrm{~nm}$.

To obtain the nanocomposite of nanosilica-polyester, the polymer polyester is mixed with cobalt and hardener. The purpose of promoters of cobalt is to speed up the curing reaction of polyester and allow them to cure at room temperature. The cobalt-based promoter helps the catalyst to start the chemical reaction between the resin and monomer and form a cured solid. The mixture was blended with particles of nano-silica at room temperature. The nanocomposite was added to molds that prepared previously to obtain samples with dimensions' length $22 \mathrm{~mm}$, width $5 \mathrm{~mm}$ and thickness of $2.5 \mathrm{~mm}$. By the same process different sample of different particle reinforcement were obtained, plain polyester, $5 \%$ particles and $10 \%$ particle reinforcement.

\subsection{Testing of Mechanical Properties}

The prepared samples were tested with using apparatus tensile test of Instron, as shown in Figure (1). For each type three samples were tested at speed rate of $0.5 \mathrm{~mm} / \mathrm{sec}$. 


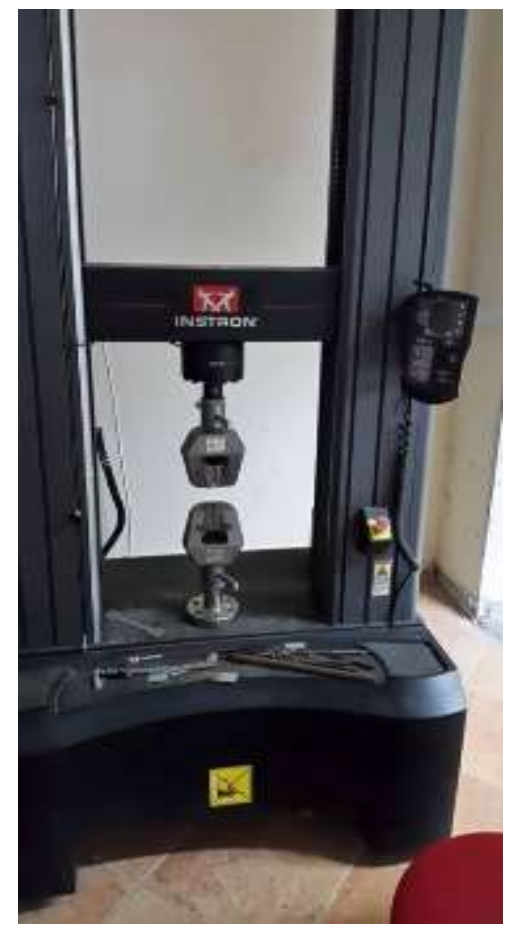

Figure (1). Tensile Test Machine.

\section{Numerical Analysis}

\subsection{Micromechanics of Composites}

The micromechanics are considered to be the heterogeneous materials with the level scale of the individual constituents. A closer inspection of any material will exhibit the degree of heterogeneity that exists. The deviation from the homogeneous state to heterogeneous exists due to several reasons, such as particles, voids, cracks and grain boundaries.

One of the main aims of micromechanics is to dermine the contribution of those factors to the macroscopic behavior of the materials and to remark the links of the mechanical relations on different length scales. As a consequence of that inhomogeneous state, on the present of a far field of load on the boundaries of the composite materials, non- homogenous distribution of stresses that developed throughout the composite materials. Evaluation of this kind of distribution, for the aim of material characterization, has always been a big challenge for engineers. One of the most popular micromechanical methods is representative volume element [19].

\subsection{Representative Volume Element (RVE)}

Computational micromechanics for material representation can be implemented under the framework of Representative Volume Element (RVE). An RVE is a method of connecting or bridging meso to micro scale. RVE is considered to be an element volume that is representative of the material structure as a whole. RVE can be seen as a small specimen and to be large enough to be representative of the macroscopic structure.

RVE can be representative if it is chosen to be large enough in order to include all sufficient information about the microstructure; however it must be much smaller than the macroscopic scale of the heterogeneous material. In this method, a unit cell will model whose properties represent the heterogeneous material as a whole, irrespective of the displacement, force or thermal loading [20], as shown in Figure (2). 


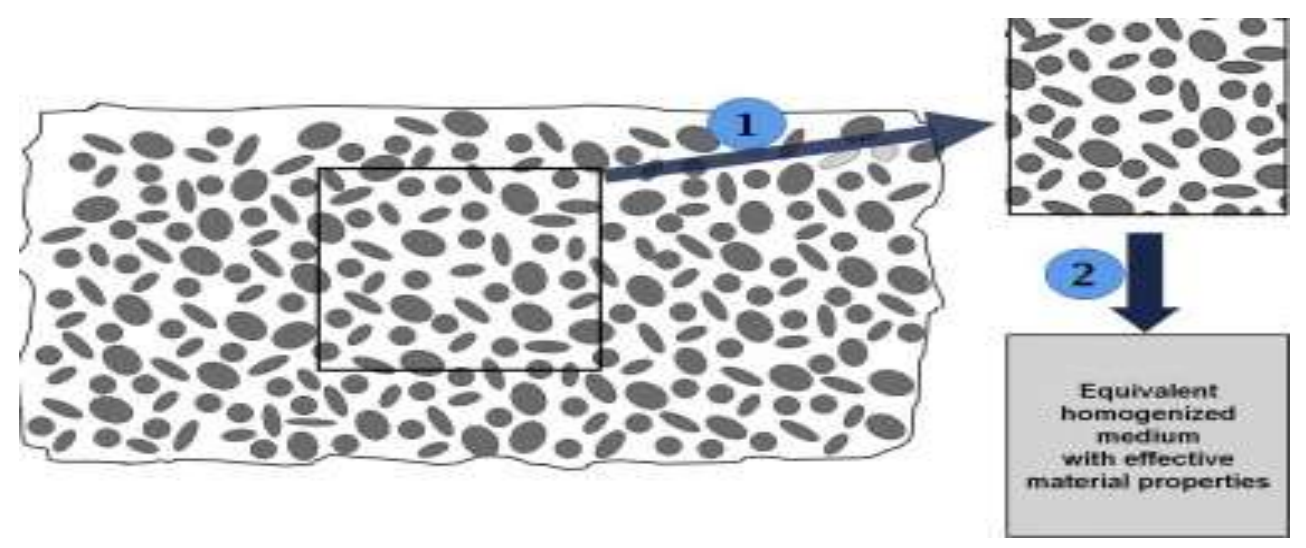

Figure (2). Representative volume element (RVE) [20].

The above steps can be summarised from macro-level strains and introduced as boundary conditions into the lower scale. At which the material is considered as heterogeneous. Then, the homogenisation process takes place and the effective properties will calculate $[21,22,23]$. The process of a multi-scale method can be seen in the schematic in Figure (3).

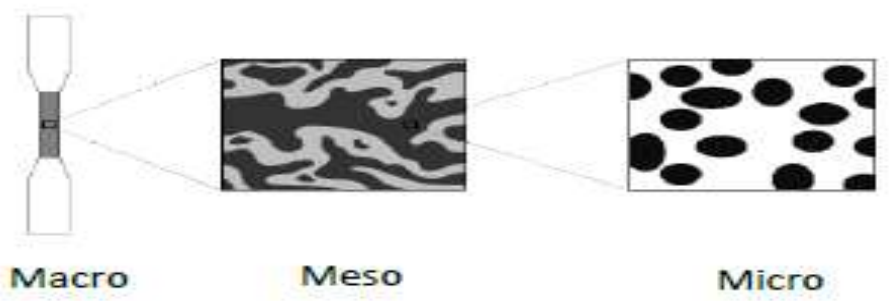

Figure (3). Representation of the approach of multi-scale [21].

\subsection{Modelling and Analysis}

Different unit cells of the nanocomposite were modelled with using Solid work software. However, Ansys workbench were used for analysing the unit cells after applying the required boundary conditions that explained in the next section. The homogenized mechanical properties of different RVE's with various volume fractions of nano silica particles content of $1 \% .5 \%, 10 \%$ and $20 \%$ were predicted.

For modelling different particles of Nano silica were reinforced with considering periodicity of the fibers, as shown in Figures (4).

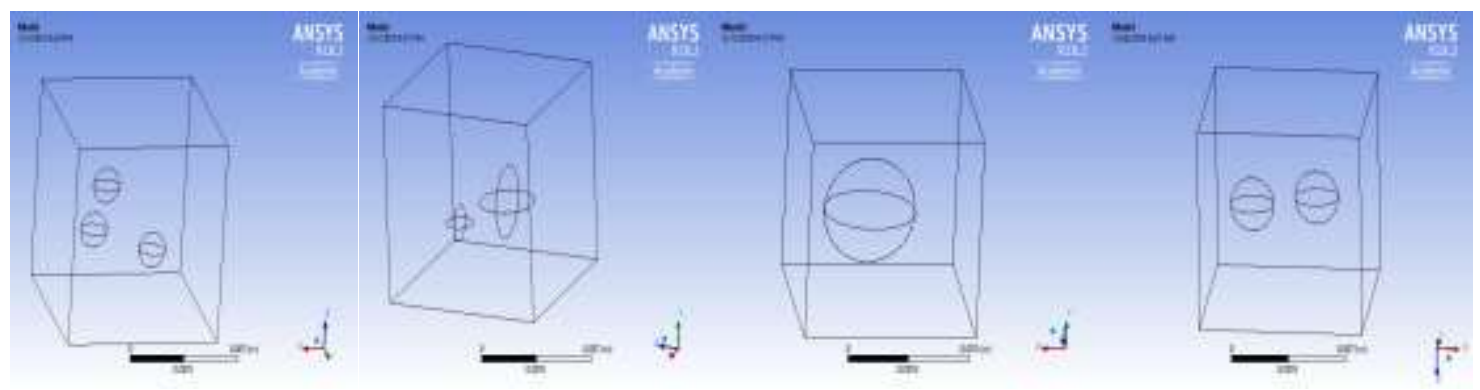

Figure (4). Compsosite reinforced with different particle inclusion with fibre content of $20 \%$. 


\subsection{Boundary Conditions}

The homogenous properties of the nano-composite were obtained by applying suitable boundary conditions to the unit cells. By fixing the faces from all direction except the length direction, as the force were applied to that direction. The way that used for evaluating the Young's modulus for the unit cells is by comparing the homogeneous unit cells. This method is dependent of the results of finite element analysis. The same method was used for the fiber-matrix RVE with different in the single homogeneous modulus. The value of the Young's modulus were changed until matching the reaction force of the heterogeneous material of the nano-composite of particle-matrix.

The meshing of the unit cells were based on the 20 node hexahedral (SOLID186) elements used for the particle and second order ten nodes of tetrahedral solid elements (SOLID187 for the matrix and. The meshing is shown in Figure (5).

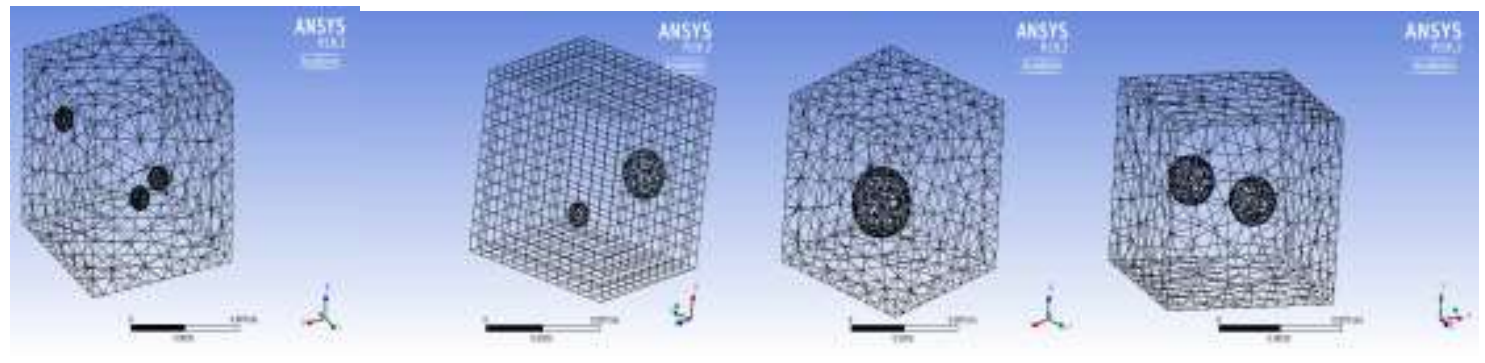

Figure (5). Unit Cells Meshing.

\section{Results and Discussions}

\subsection{Experimental Results}

Several mechanical properties were obtained from the tensile test for different kinds of samples. The tensile test result for plain polyester is shown in Figure (6).

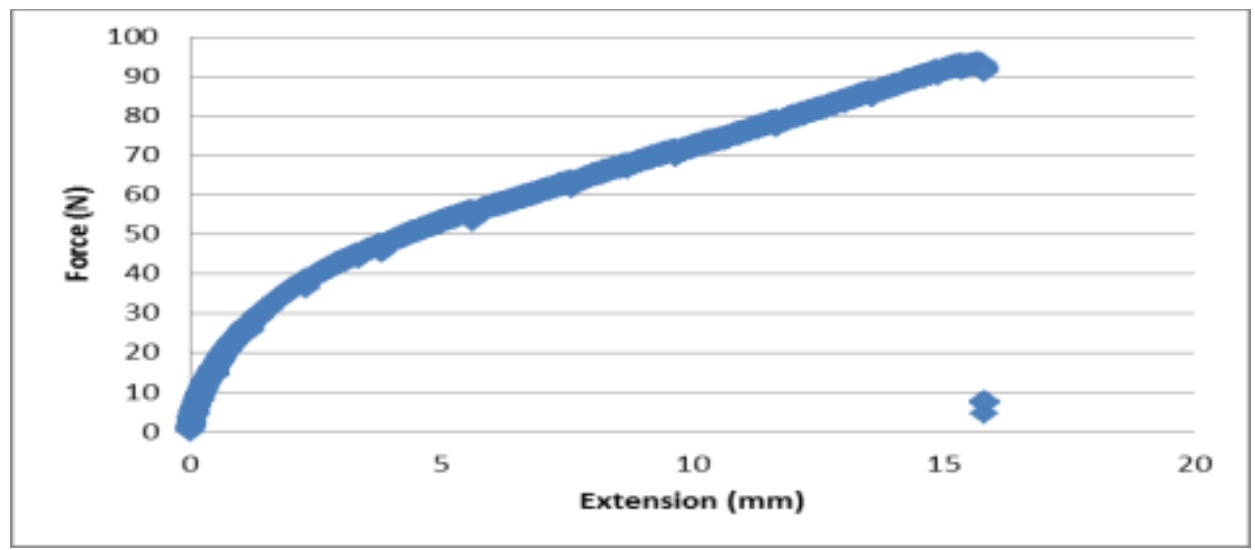

Figure (6). Tensile test result for plain polyester.

The results show a ductile behaviour of the force-extension diagram. The same test with was conducted with polyesters reinforced with different content of particles of nanosilica with values of $5 \%$ and $10 \%$ as shown in Figure (7) and Figure (8), respectively. 


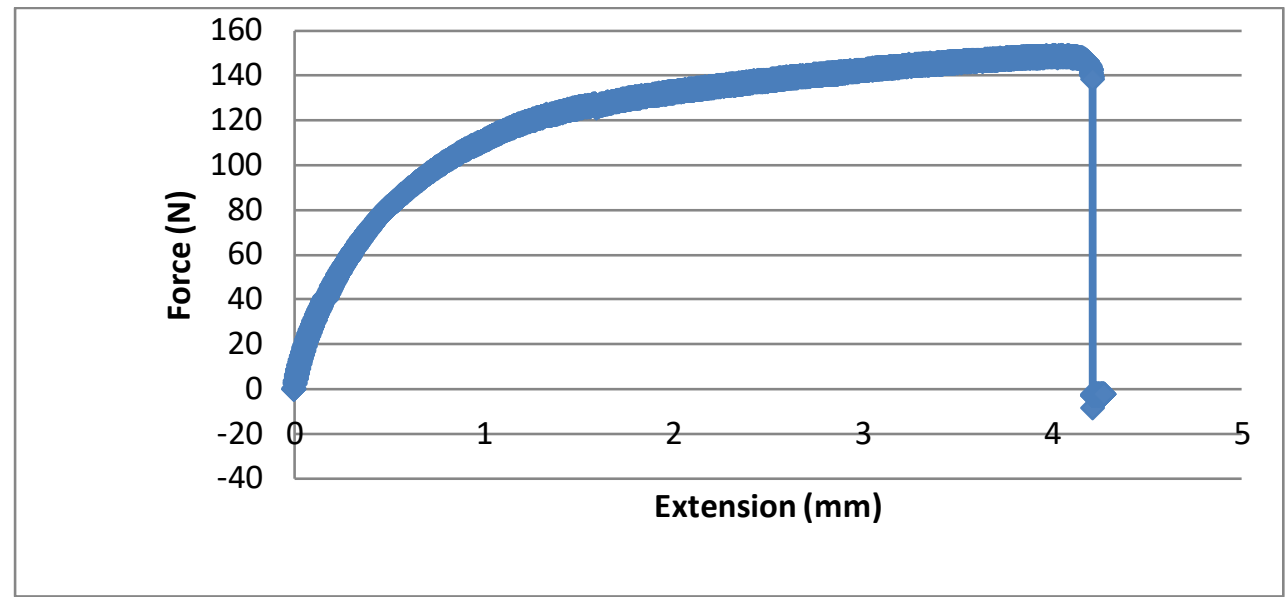

Figure (7). Tensile test for 5\% nano-silica reinforced polyester.

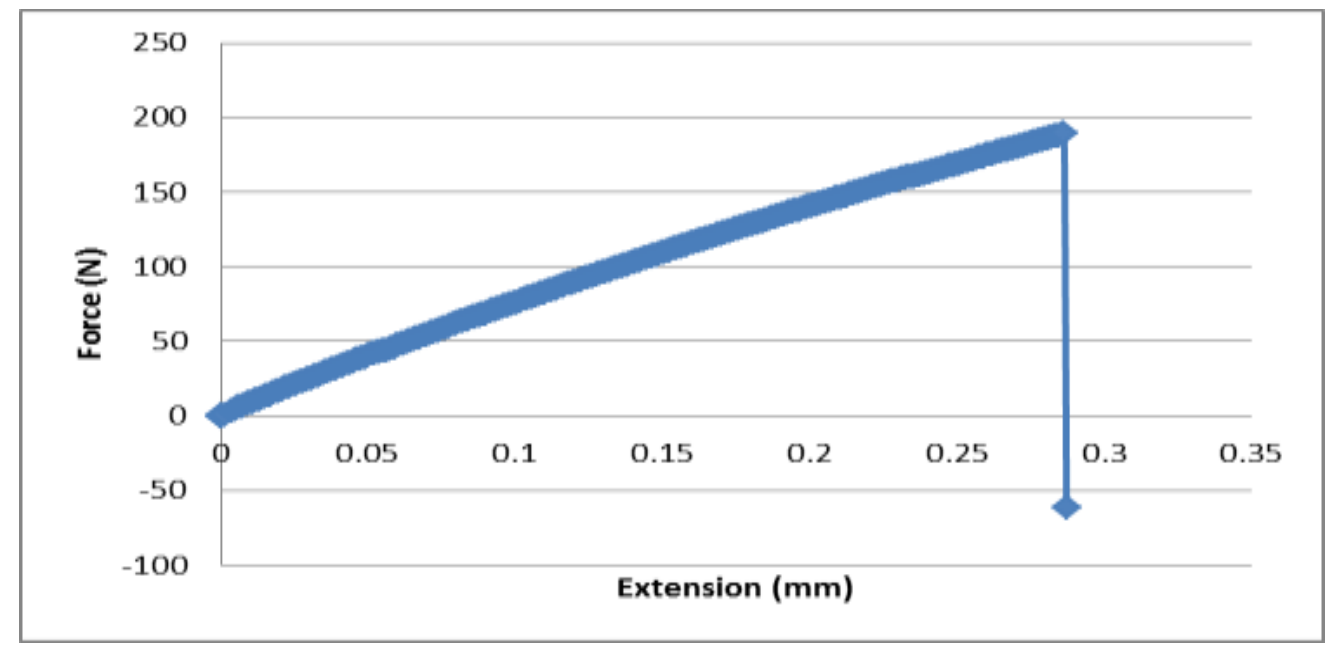

Figure (8). Tensile test for $10 \%$ nano-silica reinforced polyester.

The results show stronger behaviour of nanocomposites by comparing with plain polymer. It can be observed increasing nanocomposite stress with increasing nanoparticles ratio. However, the ductility of nanocomposites decreased by comparing with polyester, and that brittleness increased with particles content.

To conduct a comparison between the maximum stress and strain results of different particle content, Table (1) were prepared from the tensile test results.

Table (1). Comparison for polyester with various nanoparticle content.

\begin{tabular}{|c|c|c|c|c|}
\hline $\begin{array}{c}\text { Particle } \\
\text { reinforcement }\end{array}$ & Maximum force $(\mathrm{N})$ & $\begin{array}{c}\text { Maximum extension } \\
(\mathrm{mm})\end{array}$ & $\begin{array}{c}\text { Maximum stress } \\
(\mathrm{MPa})\end{array}$ & $\begin{array}{c}\text { Maximum strain } \\
(\mathrm{mm} / \mathrm{mm})\end{array}$ \\
\hline $\begin{array}{c}\text { Plain } \\
\text { polyester }\end{array}$ & 93 & 15.8 & 7.4 & 0.74 \\
\hline $\begin{array}{c}5 \% \text { particle } \\
\text { percent } \\
\text { reinforced }\end{array}$ & 148 & 4.2 & 11.5 & 0.19 \\
\hline $\begin{array}{c}10 \% \text { particle } \\
\text { percent } \\
\text { reinforced }\end{array}$ & 189 & 0.28 & 15.1 & 0.013 \\
\hline
\end{tabular}

From Table (1) it can observe that increasing particle content make the material stiffer with decrease in elongation. Also, it shows that adding $\% 5$ of Nono silica increased the force by $\% 62.8$, extension decreased by 
$\% 27$, stress increased by $\% 64$ and strain decreased by $\% 27$. However, with $10 \%$ Nano-silica content the rate was decreased.

\subsection{Numerical Results}

With using numerical analysis and RVE method, the Young's modulus for different particle content were obtained. Table 2 shows the Young's modulus that obtained for particle content of 1\%, 5\%, 10\% and $20 \%$.

Table (2). Comparison between volume fraction 0\%, 1\%,5\%, 10\% and 20\% particles content.

\begin{tabular}{|c|c|}
\hline Particle content \% & Young's modulus( GPa) \\
\hline $0 \%$ & 3 \\
\hline $1 \%$ & 3.14 \\
\hline $5 \%$ & 4.13 \\
\hline $10 \%$ & 5.51 \\
\hline $20 \%$ & 8.32 \\
\hline
\end{tabular}

From the above table it can be observe that Yong modulus of thermoset polymer of polyester increase with increasing volume fraction nano- silica particles gradually. The stress and deformation distribution on the RVE.

The experimental results and numerical results shows increase in material modulus and strength with nanoparticle content of nanaosilica by comparing with plain polymer of polyester. That increased with increasing fibre content gradually. However, that has drawn back effect on the material elongation and material ductility.

\section{Conclusions}

The work reported in this paper set out to investigate the mechanical properties of nanocomposite of nanosilicapolyester experimentally and numerically.

From this project it was concluded that increase in the volume fraction of particles cause increase in the young modulus with decrease in the ductility of the nanocomposite material. While the Young's modulus is increased of the nanocomposite, however the unit cell is become more tough and brittle. Also, when toughened and brittle increase the directional deformation and stress of heterogeneous of material matrix- particles is increased.

Moreover, it concluded that adding $\% 5$ of nano silica increased the maximum force by $\% 62.8$, extension decreased by $\% 27$, stress increased by $\% 64$ and strain decreased by $\% 27$. However, with $10 \%$ Nano-silica content the rate were decreased.

Acknowledgements: The materials studied in this paper were provided by golden Turk Company. The materials were included Polyester thermoplastic polymer and nanosilica. 


\section{References}

[1] X. Liu, "Green and facile synthesis of highly stable gold nanoparticles via hyperbranched polymer in-situ reduction and their application in Ag+ detection and separation," Polymers, vol. 10, no.42, 2018.

[2] J. Zheng, "Silica modified by alcohol polyoxyethylene ether and silane coupling agent together to achieve high performance rubber composites using the latex compounding method," Polymers, vol. 10, no. 1, 2018.

[3] D. Tzetzisa, K. Tsongasb and G. Mansourb, "Determination of the Mechanical Properties of Epoxy Silica Nanocomposites through FEASupported Evaluation of Ball Indentation Test Results" PhD Thesis, Aristotle University of Thessaloniki, Greece, 2017.

[4] M. Islam, R. Masoodi and H. Rostami , "The Effect of Nanoparticles Percentage on Mechanical Behavior of Silica-Epoxy Nanocomposites", Journal of Nanoscience, 2013.

[5] J. Zhang J., "Highly sensitive detection of melamine based on the fluorescence resonance energy transfer between conjugated polymer nanoparticles and gold nanoparticles," Polymers, vol. 10, 2018.

[6] A. Jumahata,"Tensile properties of Nano silica/epoxy nanocomposites" The University of Sheffield. United Kingdom ,2012.

[7] M.Zappalorto, A.Pontefisso and A.Fabrizi, et. Al. "Mechanical behaviour of epoxy/silica nanocomposites: Experiments and modeling," Composites Part A: Applied Science and Manufacturing, vol. 72, pp. 58-64, 2015.

[8] A. Raymond, "Introduction to the Toughening of Polymers," ACS Publication, 2000.

[9] Z. Wang, "Study on Tensile Properties of Nanoreinforced Epoxy Polymer: Macroscopic Experiments and Nanoscale FEM Simulation Prediction" The Hong Kong Polytechnic University, Hong Kong, 2013.

[10] A. Lovell, "Studies of rubber-toughened poly(methyl methacrylate): 1. Preparation and thermal properties of blends of poly(methyl methacrylate) with multiple-layer toughening particles," Journal of Polymer, vol. 34, no. 61, 1993.

[11] B. Klusemann and B. Svendsen, "Homogenization methods for multi-phase elastic composites: Comparison and benchmarks" Technische Mechanik, vol. 4, pp. 374-386, 2010.

[12] T. Mori and K. Tanaka, "Average stress in matrix and average elastic energy of materials with misfitting inclusions," Acta Metallurgica, vol. 21, pp. 571-574, 1973.

[13] J. Halpin and J. Kardos, "The Halpin-Tsai equations: a review," Polymer Engineering Science, vol. 16, pp. 344-352, 1976.

[14] S. Boutaleb, F. Zairi and A. Mesbah, et al., Micromechanics-based modelling of stiffness and yield stress for silica/polymer nanocomposites. International Journal of Solids and Structures, volume (46): 1716-1726, 2009.

[15] G. Odegard, T. Clancy and T. Gates "Modeling of the mechanical properties of nanoparticle/polymer composites," Polymer, vol. 46, pp. 553-562, 2005.

[16] M. Azmi "Modelling interaction effect of nanosilica particles on nanosilica/ epoxy composite stiffness" The University of Sheffield, UK, 2014.

[17] H. Zhang, Z. Zhang and K. Friedrich, et. Al. "Property improvements of in situ nanocomposites with reduced interparticle distance at high nanosilica content," Acta Materialia, vol. 54, pp.1833-1842, 2006.

[18] Jiawei He, Yong Wang and Yanan Wang "Elastic Property Simulation of Nano-particle Reinforced Composites," Tianjin University of Commerce, Tianjin China, 2016.

[19] Z.Wang "Study on Tensile Properties of Nanoreinforced Epoxy Polymer: Macroscopic Experiments and Nanoscale FEM Simulation Prediction," Harbin Engineering University, Harbin, China, 2013.

[20] I. Gitman, "Representative volumes and multi-scale modelling of quasi-brittle materials," Delft University of Technology, 2006.

[21] I. Ioannou, "A Study on the Numerical Characterisation of Short Fibre Reinforced Composites," The University of Sheffield, UK, 2014.

[22] M. Naffakh, and A. Díez-Pascual, " Thermoplastic polymer nanocomposites based on inorganic fullerenelike nanoparticles and inorganic nanotubes," Inorganics, vol. 2, pp. 291-312, 2014.

[23] Małgorzata Chwał and Aleksander Muc, "FEM micromechanical modeling of nanocomposites with carbon nanotubes," Reviews on Advanced Materials Science vol. 60, pp 342-351, 2021. 